\title{
All-Optical broadband phase noise emulation
}

\author{
Liam Jones*, Francesca Parmigiani, Joseph Kakande, Periklis Petropoulos, David J. Richardson \\ Optoelectronics Research Centre, University of Southampton, Southampton, SO17 1BJ, UK \\ *Imj1g10@orc.soton.ac.uk
}

\begin{abstract}
We demonstrate and characterize a technique to emulate broadband phase noise. This is achieved by exploiting cross-phase modulation induced spectral broadening, in a highly nonlinear fiber, of a signal from an intense incoherent light source.

OCIS codes: (060.2330) Fiber optics communications; (060.4370) Nonlinear optics, fibers; (060.5060) Phase modulation.
\end{abstract}

\section{Introduction}

Phase noise introduced during transmission both from amplified spontaneous emission (ASE) in optical amplifiers and nonlinear interactions between channels, mainly as a result of Kerr nonlinearity [1,2], may seriously impair modern telecommunications systems, where the signals modulate both the amplitude and phase of the optical carrier. To mimic real transmission noise and thus study the performance of processing systems, such as optical regenerators, laboratory experiments have used various noise emulation techniques. The most common one is to use ASE noise coupled with the signal to characterize [3], where both amplitude and phase noise is generated simultaneously. However, the ability to emulate amplitude and phase noise separately would prove valuable when analyzing the performance of signal processing elements. This has been achieved in the past using phase and/or amplitude modulators driven with sinusoidal waveforms [4], pseudo random bit sequence data pattern [5] or fastphotodetectors detecting ASE at their inputs [6]. The main drawback in using these techniques is that they are inherently limited by the bandwidth of optoelectronic devices, which is typically narrower than the ultrafast processes present in an optical transmission line. In this work, following [7], we present the detailed characterization of an all-optical technique that mimics the broadband phase noise generated during transmission, by artificially introducing phase noise onto the signal through cross phase modulation (XPM) in a highly nonlinear fibre (HNLF) using an ASE noise source as the pump.

\section{Experimental setup and results}

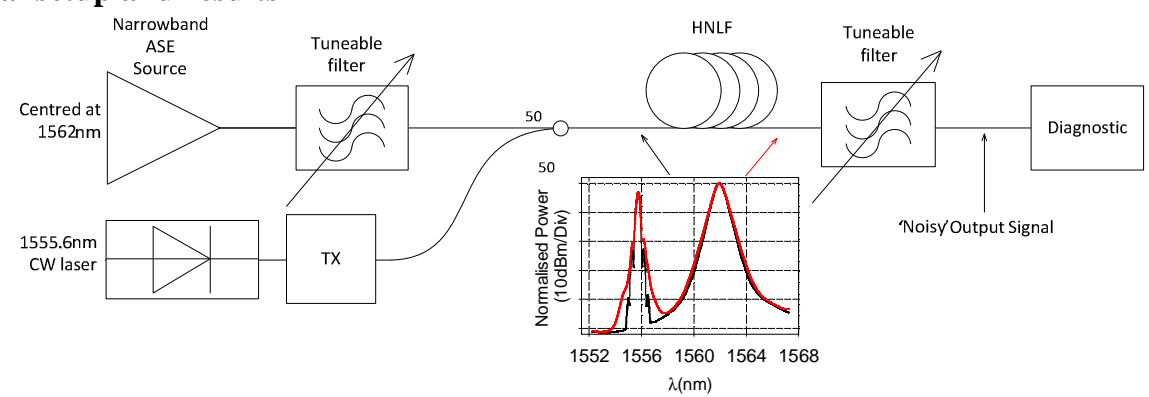

Fig. 1: Experimental setup of the all-optical XPM-based phase noise emulator. Inset: Optical spectra of 10Gbps DPSK signal and ASE pump at $11 \mathrm{dBm}$ at the input (black) and output (red) of the HNLF.

The experimental setup for the all-optical XPM induced phase noise emulator is shown in Fig. 1. A continuous wave (CW) laser source at $1555.6 \mathrm{~nm}$ was used as a carrier for a $10 \mathrm{Gbps}$ differential phase shift keying (DPSK) signal and amplified up to $8 \mathrm{dBm}$ before being combined with a narrowband ASE source, centered at $1562 \mathrm{~nm}$ and with a full width at half maximum, (FWHM) of $1 \mathrm{~nm}$. Note that the ASE bandwidth can be easily scaled to accommodate any repetition rate. The signals then co-propagated through $1 \mathrm{~km}$ length of HNLF with dispersion, dispersion slope, nonlinear coefficient and attenuation of $-17 \mathrm{ps} / \mathrm{nm} / \mathrm{km}, 0.23 \mathrm{ps} / \mathrm{nm}^{2} / \mathrm{km}, 18 \mathrm{~W}^{-1} \mathrm{~km}^{-1}$ and $2.13 \mathrm{~dB} / \mathrm{km}$, respectively. XPM causes the ASE pump amplitude variations, in the temporal domain, to be transferred onto the phase of the signal, manifested as spectral broadening. The inset of Fig. 1 shows this spectral broadening of the signal with traces displayed before (black) and after (red) the HNLF. The signal is subsequently filtered and characterized using a constellation analyzer. Firstly, as a frame of reference, the DPSK signal was characterized without any ASE noise present (referred to as 'transmitter floors' in Fig. 2). Subsequently, different levels of ASE were considered and some examples of constellation diagrams are plotted in the top line of Fig. 2. 

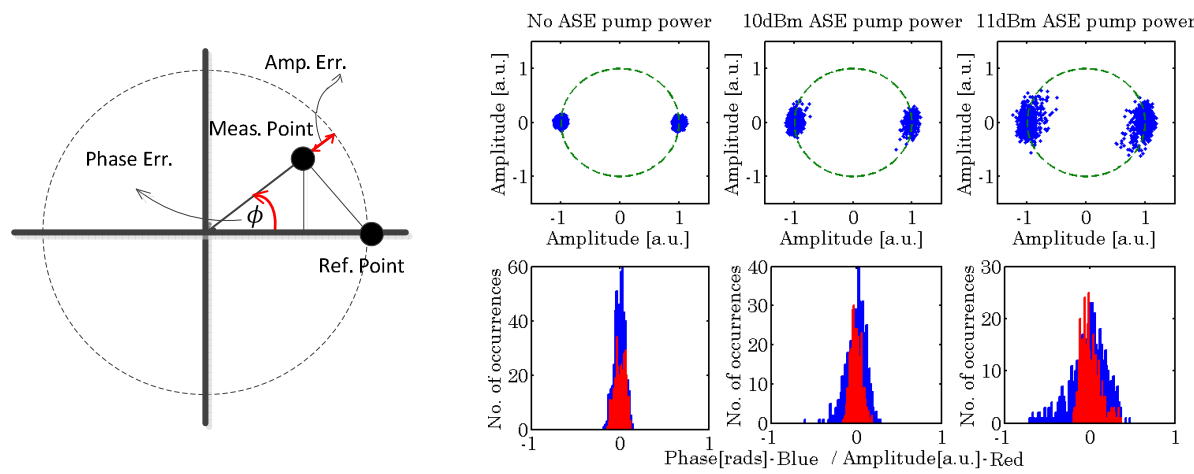

Fig. 2. Left: Method used to calculate the amplitude and phase errors of the constellation data. Upper: Constellation diagrams of the distorted $10 \mathrm{Gbps}$ signal with (from left to right) two levels of ASE pump powers, respectively. Lower: Corresponding phase (blue) and amplitude (red) histograms.

Fig. 2 (bottom) displays the corresponding histograms of the relative amplitude and phase variations for the right hand side (RHS) constellation points. Fig. 2 left, graphically displays the method used to generate the phase and amplitude error data, as seen in Fig. 3, with the reference point representing the ideal symbol and the measured point representing our sampled data point. Fig. 3, shows the phase and amplitude standard deviations as a function of the ASE power levels. Relative to the transmitter output signal, the phase standard deviation increased almost up to five times, while the amplitude noise standard deviation had only doubled. While further characterizations will be carried out, we anticipate that a more careful selection of the HNLF characteristics and the wavelength allocation of the ASE pump should further improve the results.

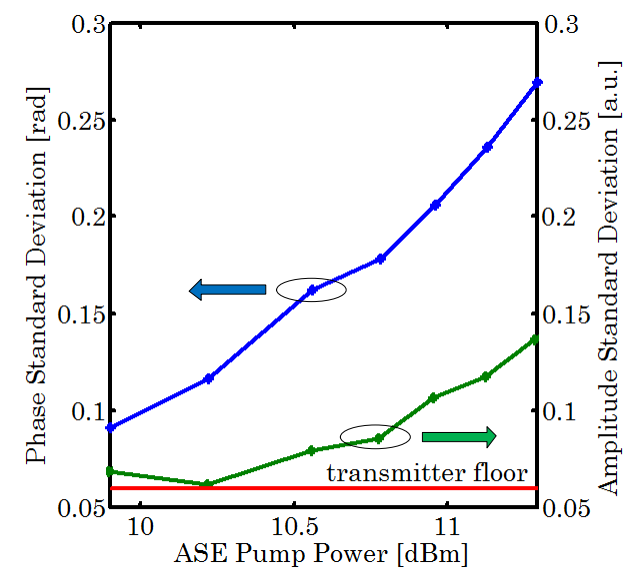

Fig.3: Standard deviations of the phase and amplitude as a function of ASE pump power, the red line corresponds to no noise added to the system.

\section{Conclusion}

We presented the characterization of a technique to introduce all-optical broadband phase noise, through XPM induced spectral broadening on a 10Gbit/s DPSK signal using a HNLF. Note that this technique is insensitive to the modulation format and repetition rate used.

\section{References}

1. J. P. Gordon and L. F. Mollenauer, "Phase noise in photonic communications systems using linear amplifiers.," Optics Letters 15, 1351-3 (1990).

2. A. Demir, "Nonlinear phase noise in Optical-Fiber-Communication Systems," Journal of Lightwave Technology 25, 2002-2032 (2007).

3. Q. T. Le, et. al. "Saturable-absorber-based phase-preserving amplitude regeneration of RZ DPSK signals," Photonics Technology Letters, IEEE 22, 887-889 (2010).

4. $\quad$ R. Slavik, et. al. "All-optical phase and amplitude regenerator for next-generation telecommunications systems," Nature Photonics 4, 690-695 (2010).

5. P. Frascella, et. al. "DPSK Signal Regeneration with a Dual-Pump Non-Degenerate Phase-Sensitive Amplifier," Photonics Technology Letters, IEEE 23 (18), 516-518 (2011).

6. J. Kakande, et. al. "QPSK Phase and Amplitude Regeneration at 56 Gbaud in a Novel Idler-Free Non-Degenerate Phase Sensitive Amplifier," Optics Communications 282, 988-991 (2009).

7. A. Mussot, et. al. "All-optical SBS reduction in fiber optic parametric amplifiers," Optics Communications 282, 988-991 (2009). 